\title{
130 nJ 77 fs Dissipative Soliton Fiber Laser
}

\author{
M. Baumgartl ${ }^{1,2}$, B. Ortaç ${ }^{1,3}$, C. Lecaplain ${ }^{4}$, A. Hideur ${ }^{4}$, J. Limpert ${ }^{1,2}$ and A. Tünnermann ${ }^{1,2,5}$ \\ 1) Friedrich-Schiller-University Jena, Institute of Applied Physics, Albert-Einstein-Str. 15, 07745 Jena, Germany \\ 2) Helmholtz-Institut Jena, Max-Wien-Platz 1, 07743 Jena, Germany \\ 3) UNAM-Institute of Materials Science and Nanotechnology, Bilkent University, 06800 Bilkent, Ankara, Turkey \\ 4) CNRS UMR 6614 CORIA, Université de Rouen, Avenue de l'Université BP 12, 76801 Saint Etienne du Rouvray Cedex, France \\ 5) Fraunhofer Institute for Applied Optics and Precision Engineering, Albert-Einstein-Str. 7, 07745 Jena, Germany \\ e-mail address: martin.baumgartl@uni-jena.de
}

\begin{abstract}
We report on ultrashort high-energy pulse generation from an all-normal-dispersion fiber oscillator. The watt-level laser directly emits chirped pulses with a duration of $1 \mathrm{ps}$ and $163 \mathrm{~nJ}$ of pulse energy. These can be compressed to $77 \mathrm{fs}$.

(C)2010 Optical Society of America

OCIS codes: (060.3510) Fiber Lasers, (140.3510) Lasers, fiber, (140.7090) Ultrafast lasers, (060.5530) Pulse propagation and solitons.
\end{abstract}

\section{Introduction}

The development of mode-locked fiber lasers operating in the normal dispersion regime to achieve higher pulse energies has been demonstrated [1]. Such lasers support dissipative solitons and could produce sub-100 fs pulses with energies as high as $30 \mathrm{~nJ}$ using standard single-mode fibers [2]. The current limitation of mode-locked stepindex single-mode fiber lasers arises mainly from high nonlinearity accumulated in small fiber core. More recently, exceptional performances in terms of pulse energy and peak power have been demonstrated in mode-locked fiber lasers using large-mode-area (LMA) photonic crystal fibers [3 and references in there]. However, these lasers operate at low $B$-integrals and produce relatively long pulses $>300 \mathrm{fs}$. In this Letter, we report the generation of sub-100 fs pulses from a passively mode-locked laser featuring a LMA photonic crystal fiber. The laser directly generates 1 ps chirped pulses with pulse energies above $160 \mathrm{~nJ}$ at a $6.15 \mathrm{MHz}$ repetition rate. The output pulses could be compressed down to $77 \mathrm{fs}$ outside the cavity. Numerical simulations show that pulse shaping in this laser is governed by both amplitude modulation by the saturable absorber mirror and nonlinear polarization rotation.

\section{Experiment and results}

The high energy passively mode-locked fiber laser is set up in a sigma cavity configuration like depicted in Fig. 1. One Key element is the Yb-doped air-clad photonic crystal fiber with $40 \mu \mathrm{m}$ core and $170 \mu \mathrm{m}$ pump cladding diameter, which is polished at an angle of $8^{\circ}$ to avoid parasitic reflections. The mode-field diameter of this fiber is about $30 \mu \mathrm{m}$. A fiber length of $1.3 \mathrm{~m}$ was chosen to obtain sufficient gain and keep nonlinearities and dispersion low at the same time. To separate the laser emission from the counter propagating pump beam a dichroic mirror is used. Self-starting passive mode-locking is achieved by employing a saturable absorber mirror (SAM) in the linear part of the cavity. The SAM bases on multi-layer GaAs/AlAs Bragg mirror and a low-temperature molecular beam epitaxy grown InGaAs quantum well structure in front of the mirror. It possesses a high modulation depth of $35 \%$, a saturation fluence of $20 \mu \mathrm{J} / \mathrm{cm}^{2}$ and a fast relaxation time of $\sim 500 \mathrm{fs}$. An optical isolator serves both as output coupler and as circulator to embed the linear part into the ring. To achieve high pulse energies already at moderate average powers and hence limit the thermal load on the SAM a confocal multipass cell is implemented in the linear segment of the cavity. This way the effective cavity length is increased to $\sim 49 \mathrm{~m}$. A set of quarter and a half wave plate is used to control the coupling ratio at the output and the nonlinear polarization evolution (NPE).

An optimization of the saturation criteria of the saturable absorber in terms of spot size resulted in stable mode-locked operation. Above threshold the laser delivers a single pulse train with a repetition rate of $6.15 \mathrm{MHz}$. Mode-locking is initiated and stabilized by the SAM, however to achieve stronger pulse shortening and thus considerable spectral broadening via self phase modulation (SPM) the wave plates had to be adjusted for significant contribution of NPE to pulse shaping. Note, that without the SAM no mode-locking was obtained at any position of the wave plates. With this optimization the laser operates in a self-starting regime at a pump power of $6.2 \mathrm{~W}$ delivering chirped output pulses of 1 ps duration at an average output power of $1 \mathrm{~W}$ possessing a pulse energy of $163 \mathrm{~nJ}$. The spectrum (Fig. 2) shows a steep edged shape which is typical for this operating regime where SPM has major influence. As a result of these steep edges the emitted pulses show small side lobes, causing a pedestal in the autocorrelation trace (Fig. 2). Assuming at least $80 \%$ of the energy in the main pulse a peak power of more than 


\section{CFK3.pdf}

$120 \mathrm{~kW}$ is accessible without external pulse compression. Using a transmission grating pair with 1250 lines / mm the pulses where compressed to near transform limited duration of $77 \mathrm{fs}$. Herein a deconvolution factor of 0.72 is used which we calculate for the transform limited pulse obtained from the experimental spectrum. The Autocorrelation trace of the compressed pulse is shown in Fig. 2 together with the autocorrelation of the transform limited pulse. Taking the side lobes into account the compressed pulses deliver a peak power of $\sim 1.2 \mathrm{MW}$, whereas due to compressor efficiency $(80 \%)$ the pulse energy is decreased to $130 \mathrm{~nJ}$. This is to our knowledge the highest peak power generated by a fiber oscillator.

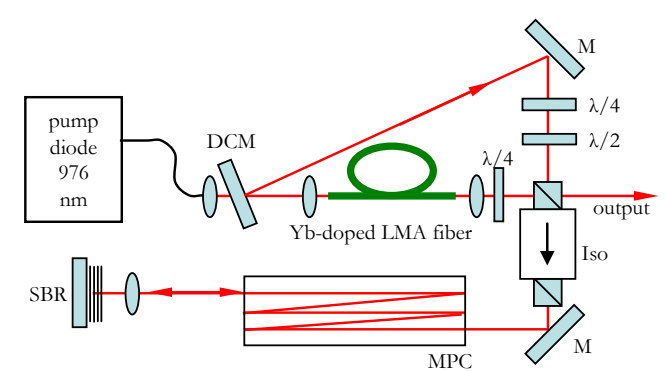

Fig. 1 Schematic representation of the passively modelocked low repetition rate $\mathrm{Yb}$-doped fiber laser, $\mathrm{M}$ : mirror, DCM: dichroic m., Iso: isolator, MPC: multi pass cell, SBR: saturable bragg reflector
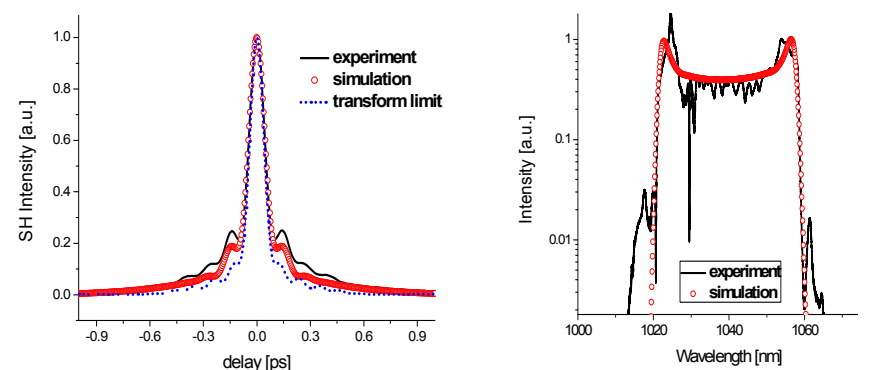

Fig. 2 Autocorrelation of compressed pulses (left) and spectrum at the laser output (right),

black/solid: experiment, red/circles: simulation, blue/dotted: transform limit

Numerical simulations based on a non-distributed model, treating every part of the cavity separately by solving the nonlinear Schrödinger equation with the split-step algorithm [4] were carried out. The experiment is well described by the model like the comparison of the simulation output with the experimental data in Fig. 2 proves. The simulation shows, that NPE is essential for pulse shaping. Temporal shortening is both provided by NPE and the SAM nonlinearity. It is well known that spectral filtering can substantially support self consistent pulse evolution. The spectral width is significantly shortened in the first part of the gain fiber, whereas it is broadened by SPM in the second. However this laser shows new pulse evolution as no additional filter element besides the gain bandwidth is needed. Low cavity dispersion together with high intracavity loss and strong amplification prevent excessive temporal broadening.

\section{Conclusion}

A passively mode-locked fiber laser is demonstrated, which produces $163 \mathrm{~nJ}$ pulses with a pulse duration of 1 ps at an average power of $1 \mathrm{~W}$ delivering a peak power of more than $120 \mathrm{~kW}$ without post processing. These pulses are compressed externally to a duration of $77 \mathrm{fs}$ at $130 \mathrm{~nJ}$ enabling peak powers of more than $1.2 \mathrm{MW}$. This performance is obtained by exploiting the combined action of a saturable absorber mirror and NPE together with a large mode area photonic crystal fiber. Pulse shaping mechanisms are studied numerically and reveal a new pulse evolution without additional spectral filter.

This work was partly supported by Helmholtz Institute Jena and Programme Inter Carnot \& Fraunhofer PICF

\section{References}

[1] A. Chong, W. H. Renninger, and F. W. Wise, “All-normal-dispersion femtosecond fiber laser with pulse energy above 20nJ," Opt. Lett. 32, 2408 (2007).

[2] K. Kieu, W. H. Renninger, A. Chong, and F. W. Wise, "Sub-100 fs pulses at watt-level powers from a dissipative-soliton fiber laser" Opt. Lett. 34, 593 (2009).

[3] B. Ortaç, M. Baumgartl, J. Limpert, and A. Tünnermann "Approaching microjoule-level pulse energy with mode-locked femtosecond fiber lasers," Optics Lett., 34, 1585, 2009.

[4] T. Schreiber, B. Ortaç, J. Limpert, A. Tünnermann, "On the study of pulse evolution in ultra-short pulse mode-locked fiber lasers by numerical simulation," Opt. Exp. 15, 8252-8262 (2007). 